\title{
MÁS ALLÁ DEL CRITICISMO RADICAL. LANGE Y LA HERENCIA KANTIANA EN NIETZSCHE ${ }^{1}$
}

\author{
Raúl Villarroel Soto ${ }^{2}$ \\ Universidad de Chile \\ rvillarr@uchile.cl \\ Daniel Pérez Fajardo \\ Universidad de Chile \\ d.perez.fajardo@gmail.com \\ Nicolás Rojas Cortés ${ }^{4}$ \\ Universidad de Chile \\ nicolas.rojas.c@ug.uchile.cl
}

\begin{abstract}
RESUMEN / ABSTRACT
El esclarecimiento y la determinación exacta de cuáles pueden haber sido las fuentes, autores o ideas que influyeron decisivamente el pensamiento de Friedrich Nietzsche y el modo como tales referencias teórico-conceptuales pudieron haber quedado incorporadas y expresadas luego en toda la extensión de su obra, constituye uno de los asuntos más complejos y difíciles de abordar para la investigación académica especializada. A materializar dicha tarea indagatoria se dirige este artículo, buscando con ello proveer una estrategia de lectura que permita visualizar focalizadamente ciertos elementos centrales de la reflexión nietzscheana que no siempre son bien reconocidos en la investigación respectiva, cuando no quedan desatendidos por completo en el corpus interpretativo que se ha venido consagrando en relación con su quehacer filosófico. Apoyándose en la investigación especializada, este artículo busca precisar el impacto que pudo haber tenido el pensamiento del filósofo F. A. Lange en Nietzsche; sobre todo reconociendo ciertos elementos de la filosofía crítica de Kant que habiendo sido inicialmente recogidos por Lange, fueron luego heredados como remanentes por Nietzsche, aunque también modificados en su obra, fenómeno que hemos denominado "criticismo radical".
\end{abstract}

Palabras Clave: Nietzsche, Lange, Kant, criticismo radical, epistemología.

El presente trabajo es resultado del Proyecto de investigación: "Pesquisa de los elementos trascendentales en la obra temprana de Friedrich Nietzsche. De la Crítica de la Razón Pura de I. Kant a la Historia del Materialismo de F. A. Lange", 2018-2019. Investigador responsable: Raúl Villarroel - Co-investigadores: Daniel Pérez, Nicolás Rojas. Proyecto financiado por el Concurso de Apoyo a la Productividad Académica en Ciencias Sociales, Humanidades, Artes y Educación (PROA) de la Vicerrectoría de Investigación y Desarrollo de la Universidad de Chile 2018.

2 Doctor en Filosofía. Profesor Titular. Académico del Departamento de Filosofía de la Facultad de Filosofía y Humanidades de la Universidad de Chile

Licenciado en Lengua y Literatura hispánica, Magíster en Filosofía, Universidad de Chile. Licenciado en Filosofía, Magíster en Filosofía, Universidad de Chile. 


\section{BEYOND RADICAL CRITICISM. LANGE AND THE KANTIAN HERITAGE IN NIETZSCHE}

The elucidation and exact determination of what may have been the sources, authors or ideas that decisively influenced the thought of Friedrich Nietzsche and how such theoretical-conceptual references could have been incorporated and expressed later in the full extent of his work, constitutes one of the most complex and difficult issues to address for specialized academic research. To materialize this research task is directed this article, seeking to provide a reading strategy that allows focalized visualization of certain central elements of Nietzschean reflection that are not always well recognized in the respective research, when they are not completely denied in the interpretive corpus that has been established in relation to his philosophical work. Based on specialized research, this article aims to clarify the impact that the philosopher F. A. Lange's thought on Nietzsche could have had; especially recognizing certain elements of the critical philosophy of Kant that having been initially collected by Lange, were then inherited as remnants by Nietzsche, although also modified in his work, a phenomenon that we have called "radical criticism"

KEYWORDS: Nietzsche, Lange, Kant, Radical criticism, Epistemology.

\section{Introducción}

$\widehat{R M}$ El esclarecimiento preciso y la determinación exacta de cuáles pueden haber sido las fuentes, autores o ideas que influyeron decisivamente el pensamiento de Friedrich Nietzsche y el modo cómo tales referencias teórico-conceptuales pudieron haber quedado incorporadas y expresadas luego en toda la extensión de su obra -tanto en aquella publicada como en aquella otra póstuma-constituye uno de los asuntos más complejos y difíciles de abordar para la investigación académica especializada. Ello, porque dicha tarea indagatoria, lejos de implicar el simple acopio de datos referenciales diversos -a menudo no mucho más que anecdóticos-, parece constituir una alternativa más que promisoria para dar lugar a lecturas críticas y quizás renovadas de su filosofía, sobre todo cuando a partir de allí se hace posible visualizar focalizadamente ciertos elementos centrales de su reflexión que no siempre son bien reconocidos en la literatura específica, cuando no quedan desatendidos por completo en el corpus interpretativo canónico que en la historia del pensamiento se ha venido consagrando en relación con su quehacer filosófico.

Teniendo lo dicho en consideración, la propuesta fundamental de este trabajo girará en torno a la posibilidad de iluminar perspectivas -a nuestro juicio- muy poco exploradas hasta ahora en la obra del filósofo alemán, por cuyo reconocimiento y presentación creemos que se puede contribuir a poner en visibilidad una dimensión problemática y ciertamente relevante de su filosofía, que podría considerarse hasta aquí más bien relegada por la hermenéutica nietzscheana tradicional.

Tal cometido será llevado a cabo a través de un trabajo que buscará explorar las lecturas de Nietzsche con el propósito de identificar y estabilizar luego en ellas el probable 
impacto que la figura y el pensamiento de Friedrich Albert Lange tuvieron en Nietzsche, buscando por esta vía avanzar hacia una expectativa dilucidadora respecto de aquellos remanentes de sello kantiano que pudieran haberse vehiculizado y sobrevivido en el pensamiento del filósofo de Sils-Maria mediante tal intermediación. Específicamente, se trataría de vislumbrar y proponer un panorama teórico en el que se consiguiera dar sentido y mayor fundamento teórico a lo que la recepción académica ha calificado como el 'criticismo radical' nietzscheano (Salaquarda 1978, 245).

\section{La herencia de Lange en el pensamiento de Nietzsche}

La temprana admiración de Nietzsche por Schopenhauer es uno de los asuntos más trabajados y respaldados investigativamente en relación con las influencias que recayeron sobre su pensamiento. Se trata de un enclave analítico ya bastante bien asentado por la crítica experta, que puede servirnos como punto de anclaje o de inicio para sostener una lectura referida a la influencia kantiana presente en la obra de Nietzsche, una vez que a ella se asocia la figura de Lange, pensador "cuyas influencias en la formación filosófica del joven Nietzsche es, sin duda, de una importancia similar a la de Schopenhauer, aunque aparezca menos explícita" (Sánchez-Meca 2011: 20). Un estudio acabado de la influencia de Lange en el pensamiento de Nietzsche-pensamos- mostraría el sendero por el que Kant podría haber hecho ingreso, incluso hasta subrepticiamente, en el universo de ideas nietzscheano. Por cierto, es razonable suponer desde ya que por ello se puede llegar a identificar, tanto en Lange como en Schopenhauer, un trasfondo conceptual claramente ajustado a los supuestos fundamentales de la epistemología kantiana.

La complejidad que se presenta a la hora de determinar las fuentes de las que se nutrió un pensador tan subversivo como Nietzsche es sin duda sustantiva, incluso habida cuenta de todo el trabajo académico desarrollado hasta la fecha. Ello, al punto de considerar que:

Langes Geschichte des Materialismus ... ist das Werk, das Nietzsche die Grundlagen seiner philosophischen Bildung gegeben hat. Überhaupt kann der Einfluß dieser Lektüre gar nicht groß genug eingeschätzt werden und geht weit über die Zeit der ersten entscheidenden Anregung (1866/68) hinaus. Zieht man einmal die persönlichen Faktoren und den Erlebniswert der Schopenhauerschen Philosophie ab, so steht für Nietzsche Lange sogar vor Schopenhauer, insofern jener auf Langes positivistischen Idealismus und Relativismus als das Fundament den Voluntarismus wie ein oberes Stockwerk aufsetzt ${ }^{5}$ (Salaquarda 1978, p. 238)

5 "La historia del Materialismo de Lange... es el trabajo que le dio a Nietzsche los cimientos de su formación filosófica. En general, la influencia de esta lectura no puede ser lo suficientemente estimada y trasciende más allá del decisivo primer estímulo (1866/68). Si por una vez se restan los factores personales y el valor de la vida de la filosofía de Schopenhauer, para Nietzsche la influencia de Lange estaría delante de Schopenhauer, 
Entonces, con el propósito de acometer una interpretación que le haga entera justicia al pensamiento nietzscheano, resulta imposible no considerar, al menos, la Geschichte des Materialismus (1877) de Friedrich Albert Lange. Para ilustrar este fenómeno creemos que vale la pena tener en cuenta las cartas que Nietzsche intercambia con sus amigos durante el periodo de su juventud, en las que se puede advertir el peso específico que ya en esos años tiene para él la figura del filósofo decimonónico alemán. En parte de esta correspondencia se deja establecido algo que no deja de resultar curioso, sobre todo si se atiende a la dependencia que entonces Nietzsche todavía mantenía respecto de su mentor, el filólogo y erudito alemán Friedrich Ritschl, para quien el pensamiento de Lange, a pesar de prestar atención a las intuiciones de la filosofía kantiana, no significa algo digno de apreciación que logre superar los rendimientos metafísicos y éticos que presenta la filosofía de Schopenhauer. Decimos que es curioso porque esta apreciación del influyente Ritschl no parece haber afectado mayormente el interés de Nietzsche por el trabajo filosófico de Lange, quien, sin duda, constituyó una pieza fundamental en su incipiente pensamiento. Considérese al respecto, por ejemplo, su carta del 20 de Julio de 1868:

...Ganz gewiß hat er (sc. Lange) Recht, mit Kants Entdeckung von der Subjectivität der Anschauungsformen so bitter Ernst zu machen, und wenn er Recht hat, ist es dann nicht ganz in der Ordnung, daß ein Jeder sich eine Weltanschauung wähle die ihm, d. h. seinem ethischen Bedürfniß, als seinem eigentlichen Wesen, genüge? Nun sagt mir eine Anschauung, die den tiefen, herben Ernst jenes gänzlich Unbekannten stark betont, innerlichst zu, und so ist mir auch durch die wachsende Überzeugung von der subjectiven Phantastik aller Speculation die Schopenhauersehe Lehre durchaus nicht im Werthe gesunken: ein Factum, das gegentheils wieder bestätigt, daß der Wille, das $\tilde{\eta} \theta o \varsigma$, stärker, primärer ist als der kühl erwägende Intellect. — Auch in diesen wichtigen Puncten stimmen wir Beide, lieber Freund, ja wol von Herzen zusammen (Salaquarda 1978:241) ${ }^{6}$.

Por otra parte, en una carta de 1866, dirigida a Hermann Mushacke, Nietzsche afirma: Kant, Schopenhauer und dies Buch von Lange-mehr brauche ich nicht ${ }^{7}$. Consideramos,

en tanto que aquel [Nietzsche] pone al idealismo y al relativismo de Lange en cuanto fundamento del voluntarismo, [y este] como un piso superior" (traducción nuestra).

6 "Ciertamente, él está en lo correcto (Lange), al tomar en serio el descubrimiento de Kant de la subjetividad de las formas de la intuición de manera tan intensa, y si tiene razón, ¿no es correcto que cada uno elija para sí una cosmovisión que sea suficiente para su necesidad ética como su propio ser? Ahora bien, una intuición que enfatiza fuertemente la profunda y severa seriedad de lo completamente desconocido me agrada de modo más íntimo, y así para mí, por la creciente convicción de la fantasía subjetiva de toda especulación, la doctrina de Schopanhauer no ha perdido valor de ningún modo: un hecho que, por el contrario, todavía confirma que la voluntad, el $\tilde{\eta} \theta$ os, es más fuerte y primario que el intelecto fríamente calculador" (traducción nuestra).

$7 \quad$ Cfr. N. B. La obra filosófica más importante que ha aparecido en los últimos decenios es sin duda la Historia del materialismo de Lange, de la que podría escribirte un montón de 
entonces, que preguntarse por la influencia que Nietzsche recibió de Lange por medio de la lectura de sus obras es un ejercicio metódicamente esclarecedor, en vistas de una comprensión acabada de su pensamiento en general, cuestión que entraña de manera bastante decisiva la posibilidad de reconocer por ese cauce el ingreso de elementos del criticismo kantiano - a menudo poco advertidos por parte de la escolaridad especializada actual- que arraigarán a modo de fundamentos en su pensamiento.

\section{Un criticismo radical: el fundamento epistemológico kantiano}

En tal sentido, y teniendo en cuenta a estas alturas la ya clásica lectura de Jörg Salaquarda (1978), no nos parece una cuestión superficial preguntarnos qué se entiende cuando se dice que Nietzsche es un pensador 'crítico radical' (1978, p. 245). Afirmamos con respecto a ello que el 'criticismo radical' debe ser comprendido como una posición filosófica que responde a una determinada interpretación de la filosofía de I. Kant, lo que no es otra cosa que decir: la interpretación de F. A. Lange. En virtud de lo cual y según lo expuesto por Salaquarda (1978), este afirmaría que todo lo que podemos llegar a conocer pertenece al mundo fenoménico, y nuestras potencias cognitivas (die Sinne und das Gehirn) juegan un papel determinante en el límite de nuestro conocimiento. Consecuentemente con esto, las interpretaciones filosóficas de la realidad no pueden comprenderse simplemente como una proyección de nuestras categorías cognitivas, sino más bien como una respuesta e interpretación, producto de nuestra fantasía, frente al mundo, a una obra de arte -como lo deja ver Lange con su noción de 'Begriffsdichtung' (Salaquarda 1978, p. 236).

Al mismo tiempo que estos preceptos son válidos para Lange, también afirma que la 'cosa-en-sí' es incognoscible en tanto que no podemos saber si el mundo de la 'cosa-en-sí' no está también condicionado por nuestra organización subjetiva. Dicho de otra manera, el pensamiento de Lange -que es el que hereda Nietzsche, según lo reafirmamos acá- es 'crítico' porque admite que nuestro conocimiento depende de nuestra constitución subjetiva y las condiciones de posibilidad del conocimiento, tal como en la Crítica de Kant, pero, al mismo tiempo es radical porque a pesar de admitir la posibilidad de algo 'en-sí', no duda en preguntarse si aquella posibilidad podría ser incognoscible para nosotros, por la misma razón de nuestro conocimiento fenoménico.

Del mismo modo que para Salaquarda, pero desde una perspectiva contemporánea, podemos considerar las palabras del filósofo estadounidense George J. Stack (1983) que ha sostenido que la influencia de Lange sobre el joven Nietzsche puede encontrarse en la incorporación que este hizo de un aparato metodológico que atendía necesariamente a la posibilidad del conocimiento del sujeto con respecto al mundo: ...Lange was his critical Forscher who taught him the importance of empirical knowledge, the value of the

páginas de elogios. Kant, Schopenhauer y este libro de Lange - no necesito más. Respuesta a la carta de Hermann Mushacke "por el 15 de octubre de 1866": I/3, 162, en Nietzsche, F. (2005) Correspondencia, vol. I (junio 1850-abril 1869). Madrid: Trotta, p. 431. 
methods of the exact sciences, who opened up the world of the independent sciences to a young philologist turned philosopher (Stack 1983, p. VI). La importancia del impacto del pensamiento de Kant en Nietzsche -se puede desprender de lo dicho por Stackmodela la aparición de fundamentos que desembocan en la conformación de un sistema orientado hacia el conocimiento y sus posibilidades, ruta epistemológica que parece iluminada en Nietzsche a través del sendero que abre para él el pensamiento de Lange.

\section{Fuentes del escepticismo y el subjetivismo nietzscheanos}

El carácter de la epistemología presente en el pensamiento de Nietzsche estaría marcado, para Stack (1983), por el fondo oscuro del escepticismo, por ese conjunto de reflexiones que vendrían dadas desde la idea de la lejanía del conocimiento humano con respecto a lo en-sí, una idea que ya había sido expresada por Lange en clara referencia a Kant. Dice al respecto Stack: "That the essence of thing transcends our knowledge plays an important role in Nietzsche's thought insofar as his skepticism concerning human knowledge is, in his early writing, based upon it" (1983, p. 196). Así, entonces, para Stack, el escepticismo de Nietzsche vendría dado a partir de una especial lectura de Kant ejercida por Lange, la que habría impactado decisivamente en las concepciones respecto de la posibilidad de conocer pergeñadas por el joven Nietzsche: ...Lange is at pains to understand Kant's theory of knowledge as leading to a form of subjectivism (Stack 1983, p. 197). No podemos afirmar categóricamente que esto sea una influencia directa de Kant, sino más bien, y siguiendo en ello a Salaquarda (1978, p. 245), sostenemos que todo esto representaría una relectura o una apropiación del sistema kantiano, y -dicho en otras palabras- lo que ya hemos presentado bajo la denominación de un 'criticismo radical'.

El impacto de la apropiación del sistema kantiano hecha por Lange puede verse reflejado en Nietzsche (2010) en el fragmento póstumo de primavera de 1884, en donde citando al autor de la Geschichte nos recuerda que: "una realidad tal como el hombre se la imagina y por la que suspira, cuando su imaginación se ve sacudida: una existencia absolutamente fija, independiente de nosotros y, sin embargo, conocida por nosotros -tal realidad no existe" (Nietzsche 2010, p. 508). En este caso, se hace necesario advertir que la afirmación de Lange apunta precisamente a la realidad que es imaginada por el hombre, pero al mismo tiempo independiente de este; no a toda realidad, dejando de este modo espacio para realidades más allá de la subjetividad humana (Salaquarda 1978, p. 244-245).

Nietzsche advierte en ese planteamiento un fundamento epistemológico con consecuencias éticas, y por ello radicaliza los puntos del subjetivismo de Lange, al considerar -como se puede apreciar en la Genealogía, por ejemplo-que el proponer una moral justificada en algo sólido, externo a la subjetividad y eterno, pero que al mismo tiempo se relacione con la subjetivad humana, es una contradicción (Salaquarda 1978, p. 245). Es por ello que Nietzsche se ve obligado a ir más allá de este 'criticismo radical', alimentando un subjetivismo que descalifica la relación del sujeto con algo 
que esté más allá de sus facultades. Nietzsche (2010) continúa su comentario a Lange señalando:

“...tal realidad no existe”. Nosotros actuamos en ella: ;pero esto no le provoca orgullo alguno a Lange! nada engañoso, cambiante, dependiente, incognoscible desea, pues, para sí mismo -éstos son instintos de seres amedrentados y que todavía están dominados moralmente: suspiran por un señor absoluto, algo amoroso, que dice la verdad-en suma, este anhelo de los idealistas es moral-religioso desde el punto de vista de los esclavos. En cambio, nuestro derecho soberano de artista podría saborear el haber creado este mundo. "sólo subjetivo", ipero yo tengo la sensación inversa: nosotros lo hemos creado! (Nietzsche 2010, p. 508).

Las disquisiciones éticas que motivan el comentario de Nietzsche (2010) tienen como trasfondo los preceptos epistemológicos de un radical subjetivismo en relación con la aproximación al mundo, es decir, una suerte de apropiación y radicalización de los conceptos kantianos ya contenidos en la obra de Lange. Este subjetivismo se presenta en Nietzsche en la idea de la inseparabilidad de lo conocido con aquello que es agregado por el individuo humano, que le impide a este acceder a un objeto puramente desnudo de subjetividad. Tal escepticismo epistemológico es revisado también por el filósofo estadounidense R. Lanier Anderson (1999) bajo la idea de un perspectivismo que determina la posibilidad de certeza del conocimiento con respecto a la verdad, según un estándar dado por el sujeto que conoce:

...Nietzsche effectively reduces truth (in the only sense in which he accepts truths) to a matter of meeting our epistemic standards. This claim requires philosophical underpinnings, which pose a significant challenge to our common sense notions. According to this stronger view, the truth about the world-and thus the way things are with the objects of our knowledge-depends on our cognitive perspectives and the epistemic standards appropriate to them $(1999$, p. 55).

Estos asuntos básicos, que revelan la formulación de una epistemología afectada en segundo término por el pensamiento kantiano, otorgan antedecentes comprensivos que -habida consideración de Lange, por cierto- aportan claridad a una lectura que ve en los planteamientos de Nietzsche una preocupación por la ciencia y el método científico.

Por su parte, el filósofo italiano Pietro Gori (2015) lee dicha preocupación por la ruptura de la relación entre causa y efecto a modo de cimientos de una teoría que atiende a la relación entre cuerpo y mente en Nietzsche (2015, p. 64). Para Gori (2015), considerar la ruptura de la necesidad del conocimiento con respecto al mundo, como un paradigma científico propiamente tal, parece algo instituido en Nietzsche bajo la idea de que sobre tal quiebre se podría fundamentar una «sicología sin alma», puramente científica (Gori 2015, p. 65).

Este último punto haría aparecer a la inconexión entre conocimiento y mundo como un principio científico básico con remanentes claramente kantianos, a los cuales les es adicionada la problemática de la suspensión entre la causa y el efecto, es decir, su no necesidad. En un sentido semejante, el investigador portugués Luis Aguiar de Sousa afirma: According to Nietzsche, space and time-relations are the subjective forms 
that bestow and appearance of necessity to the "laws of nature". The regularity of nature consists in these forms alone. Their necessity is transcendental, for they are a priori forms that we ourselves bring into nature... (Sousa 2012, p. 56). El elemento no necesario del conocimiento -es decir, lo que podemos conocer en tanto que concientes de los límites de nuestra subjetividad-, signaría la visión de las ciencias en Nietzsche como trabajo y propia factura sobre el sistema trascendental kantiano, disquisiciones epistemológicas todas estas, que responden de manera clara a la lectura subjetivista que Lange le hereda a Nietzsche por referencia a Kant.

\section{Más allá de Lange}

Sostenemos que la apropiación de los elementos trascendentales en Nietzsche responde a que este desaloja los conceptos de causa y efecto del sistema elaborado por Kant. Esto puede verse en Sobre verdad y mentira en sentido extramoral (2011) o en el parágrafo 21 de Más allá del bien y el mal (2016, p. 311). Allí es posible entender que se trata de una consecuencia clarísima de la recepción acometida por Lange respecto de Kant, en la medida en que el valor de los límites de la subjetividad se torna central en su lectura. Considerando, entonces, la transferencia de ideas que va de Lange a Nietzsche, se hace posible entender a este último cuando sostiene que toda categoría que pretenda describir el mundo debe ser consciente de su ignorancia respecto al hecho de saber qué es lo real, el criterio de certeza, el conocimiento y otras cosas similares (Nietzsche 2008, p. 102). Tal conciencia no depende -alejándose con esto de su herencia kantiana a partir de Lange- de realizar una 'crítica' de nuestra facultad de conocer, ya que representa un contrasentido criticar el único instrumento que tenemos para conocer, apelando a este mismo como criterio de validación del conocimiemto.

De esta manera, a su escepticismo frente a las categorías de causa y efecto, Nietzsche agrega también los conceptos de 'sujeto'y 'objeto'. Toda categoría representa una expresión de señorío humano, es decir, un significar más que un determinar la realidad. Esta crítica metodológica se puede ver bien claramente reflejada en la interpretación del mecanicismo de Bošković o Teichmüller, que Nietzsche hereda de Lange, y que expone dentro de sus apuntes para la Voluntad de Poder: ...Una traducción de este mundo de efectos a un mundo visible - a un mundo para los ojos-es el concepto de «movimiento». En esta traducción se subentiende siempre que algo es movido -con lo cual, que sea en la ficción de un átomo-grumo o incluso de su abstracción, en el átomo dinámico, se sigue pensando aqui en una cosa que produce efectos-, es decir, no hemos salido de la rutina hacia la cual nos encaminan los sentidos y el lenguaje. Sujeto, objeto, un agente del hacer, el hacer y lo que el hacer hace, separados: no olvidemos que esto es una mera semiótica y que no designa nada que sea real. La mecánica como teoría del movimiento es ya una traducción al lenguaje de los sentidos del ser humano (2008, p. 533).

En 1888, la conciencia de que todo producto conceptual que tuviera la intención de explicar el mundo se nos presente al modo de la representación dependiente de nuestra fisiología, ya está puesta en marcha teórica. Ahora bien, si se tiene en mente 
que para Nietzsche -como hemos visto en el punto anterior- el conocimiento se comprende como algo concerniente a las relaciones y no a las esencias, se entiende que su crítica al mecanicismo no apunta a los resultados positivos que pueda tener dicha propuesta científica (Salaquarda 1978, p. 248), sino que más bien a la inconsciencia de sus presupuestos -la 'cosa en sí', el 'sujeto' o el ‘objeto', por ejemplo. Por ello, y ya separándose del 'criticismo radical' de Lange, Nietzsche propone eliminar la distinción idealista entre mundo fenoménico y 'cosa en sí', como bien refleja el archiconocido fragmento póstumo 7 [60] de finales de 1886 - primavera de 1887: Contra el positivismo, que se queda en el fenómeno "sólo hay hechos», yo diría, no, precisamente no hay hechos, sólo interpretaciones. No podemos constatar ningún factum «en sì»: quizás sea un absurdo querer algo así. "Todo es subjetivo», decís vosotros: pero ya eso es interpretación [...] (Nietzsche 2008, p. 222). La totalidad de conceptos interpretativos del mundo ganan, para Nietzsche, valor epistémico, solamente en tanto que la teoría se comprenda a sí misma como una interpretación y además sea consciente de sí como tal. Por ello es que, para Nietzsche, la ciencia en su metodología investigativa no puede sino intentar verificar los resultados de sus experimentos por medio de la comprensión de sus presupuestos.

La eliminación de la causa y el efecto del sistema trascendental kantiano se condice con una visión que sustenta al conocimiento como una subjetividad que condiciona el producto del acercamiento al mundo, premisa que, según Aguiar de Sousa (2012) emana desde la lectura de Schopenhauer; mas, esta parece responder también a las preocupaciones atendidas por Nietzsche respecto del pensamiento de Lange. Así es como el 'criticismo radical' de Lange encuentra su mayor rendimiento interpretativo a través de la lectura de Nietzsche, en cuanto que la investigación de la realidad no puede ser sino la comprensión de nuestra imposibilidad de un conocimiento seguro. Tal posición encuentra correspondencia con la lectura que hace Gori (2015) de una ciencia fundamentada sobre la base de la no coincidencia entre cuerpo y mente, sujeto y mundo, planteando a la epistemología sobre la base de dicho desacuerdo y no sobre la tentativa de su restablecimiento. Las nociones críticas que, desde Lange, alcanzan al pensamiento de Nietzsche parecen devenir en su interior en un pensamiento científico que busca ahondar en dicha diferencia, en ese contexto donde la fisiología y el materialismo parecen puntos de partidas insuficientes en tanto subjetivos a la vez que necesarios, siendo dicha subjetividad la única certeza.

\section{Conclusión}

Hasta aquí -siguiendo a la crítica académica para ello- hemos aventurado las líneas decisivas para bosquejar un proyecto hermenéutico que ayude a desvelar y determinar con precisión las fuentes y estímulos intelectuales de los que bebió Nietzsche en la instancia de la gestación de la critica radical que dirige a toda la filosofia de su época. Las mentadas líneas responden a la pregunta que subyace a nuestra investigación: ¿En qué sentido se puede afirmar que el pensamiento de Nietzsche se enlaza con el de F. A. Lange? Nuestra respuesta tanteaba una posible investigación desde tres aristas: La relación ineludible de Nietzsche con Lange se podría entender en tanto que (1) lector, 
es decir, como simplemente heredero de su pensamiento ('criticismo radical'), también como (2) intérprete, y con esto entendemos la apropiación de algunos elementos teóricos fundamentales (por ejemplo, la noción de subjetividad kantiana en cuanto principio epistemológico), pero, además (3), quizás la más importante de todas, como opositor de Lange, a la hora de hacerse consciente del peligro ético en el que deviene una epistemología heredera del sistema kantiano.

Así, entonces, se hace imposible negar que el pensamiento de Lange no esté presente en Nietzsche, en cualquiera de las tres posibilidades señaladas recién. Y no solamente en su etapa de juventud, como podría ser el caso de las motivaciones schopenhauerianas que se constatan fehacientemente en su obra y que se van desvaneciendo luego del Nacimiento de la Tragedia. En efecto, si consideramos el clásico parágrafo 21 de Más allá del Bien y el Mal, en donde se afirma que: Somos únicamente nosotros los que hemos fabulado las causas, la sucesión, la reciprocidad, la relatividad, la coacción, el número, la ley, la libertad, el fundamento, la finalidad; y si fabulamos este mundo de signos como «en sí» y lo entremezclamos con las cosas, entonces volvemos a actuar del modo en que siempre hemos hecho, a saber, mitológicamente (2016, p. 311 ), podemos observar a un Nietzsche opositor de Lange, en la medida en que el 'criticismo radical' queda superado porque él no se permite a sí mismo declarar una ingenuidad teórica de semejante envergadura como sería postular la posibilidad de un "en sí" más allá de nuestra subjetividad, menos aun cuando, al mismo tiempo, se propone que las categorías con las que el pensamiento científico se hace con el mundo responderían al actuar como siempre lo hemos hecho, es decir, desde la fisiología de nuestros sentidos y siendo que estas categorías dependen y no se pueden sino entender como consecuencias de nuestra organización subjetiva. Es de esta manera, entonces, que el escepticismo de Lange pervive - pero transformado- en la lectura de Nietzsche, y se convierte finalmente en un método de análisis de los presupuestos que nutren los sistemas epistemológicos que rigen el mundo.

Siguiendo esta línea metodológica en el quehacer filosófico de Nietzsche, podemos proyectar nuestra investigación a la actividad más propia del pensador, la crítica a la moral de la decadencia. Denominar a Nietzsche como un pensador crítico no puede simplemente querer decir que era un pensador viperino. El concepto crítico, como hemos buscado mostrarlo, nos lleva a considerar a Nietzsche un pensador mucho más kantiano de lo que él mismo se atrevería a afirmar. Es por esto que la crítica a la moral de la decadencia cobra rigor comprensivo cuando se entiende que el análisis de la moral es al mismo tiempo un análisis de los presupuestos epistemológicos y ontológicos desde los que se fundamente aquella moral. Concebimos que una hermenéutica aplicada a la reacción de Nietzsche frente a la obra Die Arbeiterfrage in ihrer Bedeutung für Gegenwart und Zukunft (1865) de F. Lange sería clarificadora para comprender los fundamentos epistemológicos del método genealógico de nuestro autor y constituiría la proyección obligada de la investigación que aquí hemos delineado en su figura inicial.

En el mismo sentido, dejamos pendiente por ahora determinar la influencia precisa de los sistemas científicos que se encuentran en la Geschichte des Materialismus (entiéndase R. Boscovich, o Teichmüller, entre otros) en función de determinar si las propuestas del Eterno Retorno y la Voluntad de Poder efectivamente responden a una 
reformulación metódica del criticismo radical de Lange, nuevamente cruzado por la interpretación de Kant, o si bien podrían haber incluso más fuentes que dejen entrever de manera clara a qué inquietudes filosóficas apuntaba Nietzsche en toda su filosofía, y afirmamos que toda su filosofía, porque con el trabajo que hemos realizado hasta aquí se hace difícil delimitar periodos estancos en la obra de nuestro pensador.

\section{Referencias bibliográficas}

Aguiar de Sousa, L. (2012), "Knowledge, truth, and the thing-in-itself: the presence of Schopenhauer's transcendental idealism in Nietzsche's on truth and lie in an extra-moral sense", As the spider spins. Essays on Nietzsche's critique and use of language. Berlin: de Gruyter; pp. 39-61.

Lanier Anderson, R. (1999), "Nietzsche's views on truth and the kantian background of his epistemology", Nietzsche, epistemology and philosophy of sciences. Nietzsche and the science II. Berlin: Springer Science+Business Media; pp. 47-59.

Gori, P. (2015), "Posizioni ottocentesche sul rapporto corpo-mente: Lange, Mach, Nietzsche", Intersezioni 1: 63-88.

Nietzsche, F. (2005), Correspondencia, vol. I (junio 1850-abril 1869). Madrid: Trotta. Tecnos. (2008), Fragmentos Póstumos. Volumen IV (1885-1889). Madrid: (2010), Fragmentos Póstumos. Volumen III (1882-1885). Madrid: Tecnos.

(2011), "Sobre verdad y mentira en sentido extra moral", Obras completas. Volumen I. Escritos de juventud. Madrid: Tecnos.

(2016), "Más allá del bien y del mal", Obras completas. Volumen IV. Escritos de madurez II. Madrid: Tecnos.

Salaquarda, J. (1978). "Nietzsche und Lange", Nietzsche-Studien $\mathrm{N}^{\circ}$ 7, volume 7, issue 1: 236-260.

Sánchez-Meca, D. (2011). “Introducción al volumen I: La evolución del pensamiento de Nietzsche en sus escritos de juventud". Nietzsche, Friedrich. Obras completas. Volumen I. Escritos de juventud. Madrid: Tecnos.

Stack, G. J. (1983), Nietzsche and Lange. Berlin: de Gruyter. 